\title{
Case Report \\ Poorly Differentiated Uterine or Cervical Sarcoma in a Young Dog
}

\author{
Michelle C. Cora ${ }^{1}$ and Jennifer A. Neel ${ }^{2}$ \\ ${ }^{1}$ Cellular and Molecular Pathology Branch, NIEHS/NIH, 111 Alexander Drive, MD B3-06, Research Triangle Park, NC 27709, USA \\ ${ }^{2}$ Department of Population Health and Pathobiology, College of Veterinary Medicine, North Carolina State University, \\ 1060 William Moore Drive, Raleigh, NC 27607, USA
}

Correspondence should be addressed to Jennifer A. Neel, jennifer_neel@ncsu.edu

Received 10 May 2011; Accepted 31 May 2011

Academic Editors: M. Bugno-Poniewierska, K. Hittmair, and J. S. Munday

Copyright (๑) 2011 M. C. Cora and J. A. Neel. This is an open access article distributed under the Creative Commons Attribution License, which permits unrestricted use, distribution, and reproduction in any medium, provided the original work is properly cited.

A 1.5 year old, female, spayed, Labrador retriever with a history of three abdominal surgeries within the previous two months presented to the North Carolina State University Veterinary Teaching Hospital for evaluation of a pelvic inlet mass causing fecal tenesmus, obstipation, and dysuria. Abdominal ultrasound revealed a caudal abdominal mass extending into the pelvic cavity. Cytologic evaluation of the mass showed a pleomorphic round to fusiform cell population with histiocytic and suppurative inflammation. The primary differential was neoplasia, but inflammation with cellular pleomorphism could not be excluded. Via histopathology and immunohistochemistry, a diagnosis of poorly differentiated sarcoma originating from the uterus or cervix with widespread intra-abdominal dissemination and metastasis was made. Sarcomas of any type are rare in young dogs with only sporadic cases of poorly or undifferentiated sarcomas reported. This case is a unique presentation of an aggressive, poorly differentiated sarcoma arising from the cervix or uterus in a young dog and illustrates the importance of histologic evaluation of surgically resected tissues that are abnormal in appearance.

\section{Introduction}

Sarcomas are rare in young dogs with few reported cases of poorly differentiated sarcomas. This paper describes a young dog with an aggressive, pleomorphic, poorly differentiated sarcoma, with widespread intra-abdominal dissemination and multiorgan metastasis.

\section{Case Presentation}

A 1.5 year old female spayed Labrador retriever presented for evaluation of a pelvic inlet mass causing fecal tenesmus, obstipation, and dysuria. Two months prior, she had presented to the referring veterinarian for bloody vaginal discharge. An open pyometra was diagnosed and an ovariohysterectomy performed. One week later, the discharge continued, and dysuria and tenesmus were also present. Vulvar swelling and serosanguinous discharge were noted, and rectal palpation revealed a painful swelling at the ventral aspect of the colon. Radiographs showed a soft tissue mass in the caudal mid-abdomen. Uterine stump pyometra was suspected. During an exploratory laparotomy, an enlarged, inflamed uterine stump was found; tissue was removed to the point of the cervix, but it was not submitted for histopathology. A urinary catheter was passed without difficulty. After surgery, serosanguinous vulvar discharge, dysuria, and tenesmus continued and a ventral colon mass was still palpable. Over the next 6 days, a urinary catheter was intermittingly placed, and enemas were administered as needed. On day 7 , abdominal radiographs revealed a markedly impacted colon, and an enema was done under anesthesia. On day 9, repeat radiographs showed a large radiopaque structure in the distal colon. During a second exploratory laparotomy, a large, hard fecolith was removed from the colon. Her clinical signs continued postoperatively and referral to the North Carolina State University, Veterinary Teaching Hospital (NCSU-VTH) was recommended.

At presentation at the NCSU-VTH, she was found to be $5 \%-7 \%$ dehydrated based on physical exam, febrile $\left(103.5^{\circ} \mathrm{F}\right)$, and dysuric with a painful distended abdomen and large bladder. Red-brown mucoid vulvar discharge was observed. A CBC revealed a moderate leukocytosis 


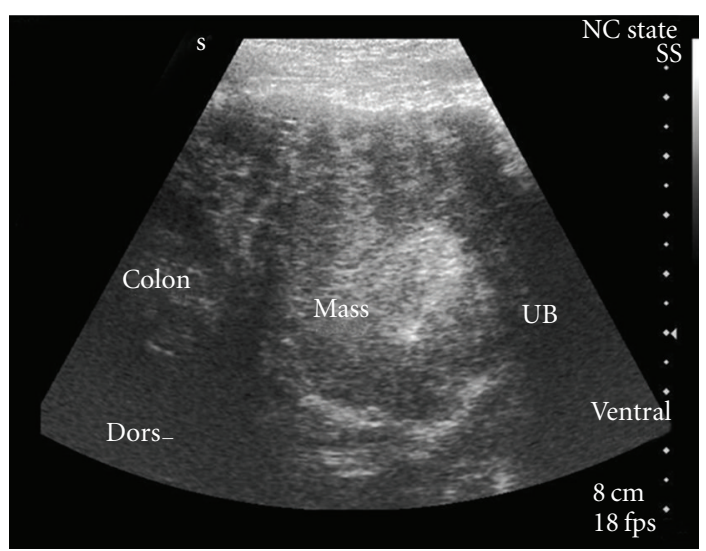

Figure 1: Abdominal ultrasound image from a young dog with a large caudal abdominal mass between the colon and the urinary bladder (UB). Primary differential diagnosis was a granuloma or neoplasia.

$\left(27.01 \times 10^{3} / \mu \mathrm{L}\right.$; reference interval $\left.4.88-12.74 \times 10^{3} / \mu \mathrm{L}\right)$ with a mature neutrophilia $\left(21.07 \times 10^{3} / \mu \mathrm{L}\right.$; reference interval $\left.2.53-12.88 \times 10^{3} / \mu \mathrm{L}\right)$ and a moderate monocytosis $(4.86 \times$ $10^{3} / \mu \mathrm{L}$; reference interval $\left.0.21-1.05 \times 10^{3} / \mu \mathrm{L}\right)$, consistent with inflammation, and mild hypoproteinemia $(5.8 \mathrm{~g} / \mathrm{dL}$; reference interval 6.1-7.5 g/dL).

A serum chemistry panel showed the following abnormalities: Mild hyperphosphatemia $(6.6 \mathrm{mg} / \mathrm{dL}$; reference interval 2.5-5.6 mg/dL), slight hypomagnesemia $(1.5 \mathrm{mg} / \mathrm{dL}$; reference interval $1.8-2.5 \mathrm{mg} / \mathrm{dL}$ ), and mild hypoproteinemia $(4.6 \mathrm{~g} / \mathrm{dL}$; reference interval $5.2-7.3 \mathrm{~g} / \mathrm{dL})$ due to hypoalbuminemia $(2.3 \mathrm{~g} / \mathrm{dL}$ reference interval $3-3.9 \mathrm{~g} / \mathrm{dL})$. Results of a urinalysis were unremarkable and urine culture was negative. The hypoproteinemia and hyoalbuminemia were attributed to the severe peritoneal fluid effusion. Potential causes for the hyperphosphatemia include young age, hemolysis and decreased glomerular filtration rate (prerenal), although urea nitrogen and creatinine were within the reference interval. The hypomagnesemia was attributed to the hypoproteinemia.

Abdominal ultrasound showed a caudal abdominal mass extending into the pelvic cavity between the colon and urinary bladder (Figure 1). Differential diagnosis included a granuloma or neoplasia. The medial iliac and hypogastric lymph nodes were enlarged and differentials included infection/inflammation, reactivity and, less likely, neoplasia. Severe peritoneal fluid accumulation and a heteroechoic mesentery were also seen. The peritoneal fluid was serosanguinous with a cell count of $12.93 \times 10^{3} / \mu \mathrm{L}$ and a protein of $3 \mathrm{~g} / \mathrm{dL}$ (exudate). Cytological examination of a cytocentrifuged preparation of the fluid revealed $2 \%$ small mononuclear cells, 11\% large mononuclear cells, $84 \%$ nondegenerate neutrophils, and 3\% eosinophils together with a reactive mesothelial cell population. No etiologic agents or neoplastic cells were identified. Aspirates of the caudal abdominal mass were taken and submitted for cytological interpretation (Figures 3 and 4).
The smears had good cellularity with focal areas of an eosinophilic stippled background. A mildly to moderately pleomorphic mononuclear cell population was seen individually and in loose clusters. Cells exhibited mild-tomoderate anisocytosis and anisokaryosis with a variable $\mathrm{N} / \mathrm{C}$ ratio. They were round to oblong to occasionally fusiform in shape with variably distinct borders. Cytoplasm was moderate, and basophilic and occasionally contained discrete vacuoles and/or blue pigment. Nuclei were round to oblong to reniform and eccentric with finely stippled chromatin and 0-3 indistinct round to oval nucleoli each. Occasional unremarkable mitotic figures and activated macrophages were also present; macrophages often contained a small amount of similar basophilic pigment. Neutrophils were mildly increased. The sample was interpreted as an atypical round to fusiform cell population with evidence of histiocytic and suppurative inflammation. The primary differential diagnosis was neoplasia (histiocytic sarcoma, other sarcomas, or atypical lymphoma); however, given the patient's age and three recent surgeries, a chronic inflammatory lesion, such as a granuloma, with marked cellular pleomorphism could not be excluded.

Due to a poor prognosis, she was humanely euthanized. On gross postmortem examination, a $6 \mathrm{~cm}$ diameter cranial vaginal mass was palpable. Greater than 2 liters of redbrown abdominal fluid was present. A $20 \mathrm{~cm}$ diameter, multilobulated, white-tan mass almost entirely effaced the cervix and was adherent to, and enveloped part of, the distal colon, both ureters, the caudal poles of both kidneys and the dorsal aspect of the urinary bladder (Figure 5). The mucosal surfaces of the ureters and bladder were normal. Many pinpoint to $1 \mathrm{~cm}$ diameter, white-tan plaques were present on the omentum, mesentery, abdominal wall, right lateral liver lobe, and diaphragm. The serosal surface of the entire gastrointestinal tract was granular, and mottled dark red, yellow, and tan, consistent with peritonitis. Scattered throughout all lung lobes were numerous white, soft nodules up to $1 \mathrm{~cm}$ in diameter. Marked enlargement of the iliac and sublumbar lymph nodes and moderate enlargement of the tracheobronchial and mediastinal lymph nodes were noted.

Histologically, the mass was an unencapsulated, infiltrative, multilobulated neoplasm composed of sheets, cords, and interlacing streams of pleomorphic spindle to round cells separated by a variably thick, fibrous stroma with multiple areas of necrosis and hemorrhage (Figure 2). Cells had distinct borders with round to fusiform nuclei, finely stippled to densely packed chromatin and up to three magenta nucleoli each. Cytoplasm ranged from elongated and fibrillar to scant and eosinophilic. Neoplastic foci were present within the colon, heart, lungs, mesentery, diaphragm, unilateral adrenal gland, and multiple lymph nodes. Immunohistochemical staining was performed. The neoplasm was strongly positive for vimentin, and negative for cytokeratin, desmin, smooth muscle actin, myoglobin, CD18, and lysozyme. Based on histopathology and immunohistochemistry results, a diagnosis of a poorly differentiated sarcoma originating from the uterus or cervix with widespread intra-abdominal dissemination and metastasis was made. 


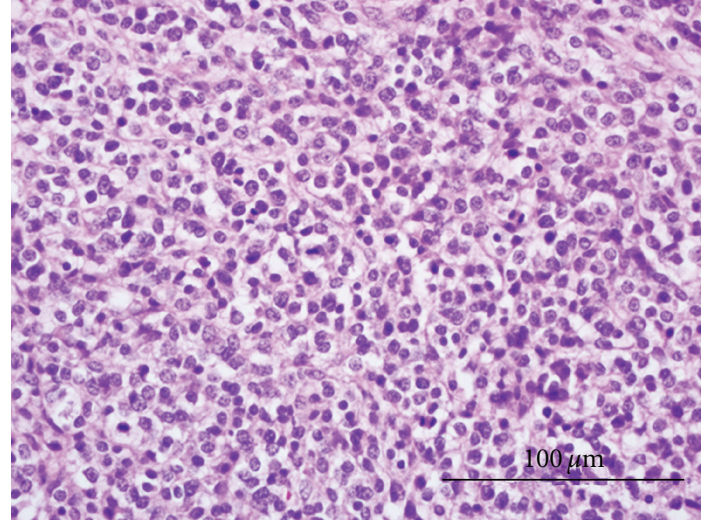

Figure 2: Histological section of a poorly differentiated sarcoma. Neoplastic cells are round to fusiform and display moderate pleomorphism. Hematoxylin and eosin stain, 40x objective, bar = $100 \mu \mathrm{m}$.

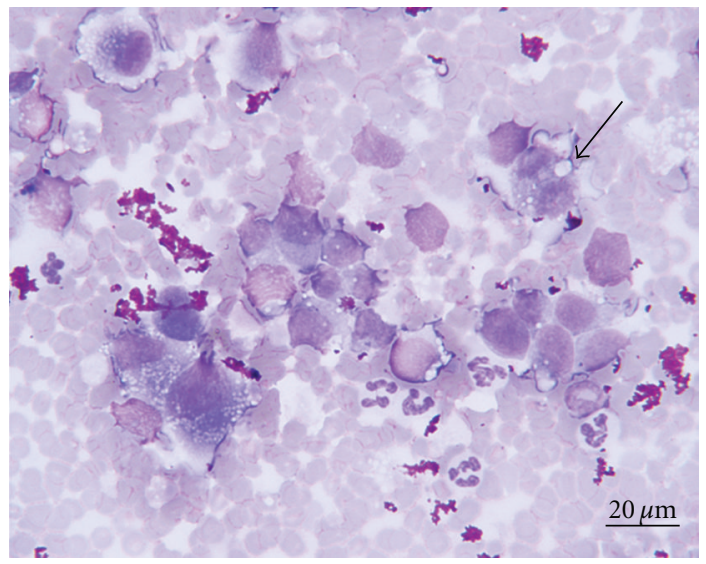

FIGURE 3: Fine-needle aspirate of an intra-abdominal mass in a young dog. Many individualized, large, round to fusiform cells that often contain vacuoles and display moderate anisocytosis and anisokaryosis are present. Note the mitotic figure (arrow). The primary differential diagnosis was neoplasia (histiocytic sarcoma, other sarcomas, or atypical lymphoma). Wright's_-Giemsa stain, $50 \mathrm{x}$ objective, bar $=20 \mu \mathrm{m}$.

\section{Discussion}

This patient had a very complicated medical history upon presentation to the NCSU-VTH. Although unproven, it is plausible that the sarcoma was present at the time of the initial and/or subsequent surgeries and that the surgeries may have contributed to the widespread intraabdominal dissemination (abdominal sarcomatosis). It was noted during the third and final surgery that the omentum and bladder were speckled and had a granular appearance to their serosal surfaces. The clinical interpretation of these findings at the time of surgery is unknown but may have been construed as inflammatory changes resulting from the previous two surgeries. It is also possible that these abnormalities were neoplastic foci. Microscopic examination (cytology or histopathology) of the tissue would have been necessary to

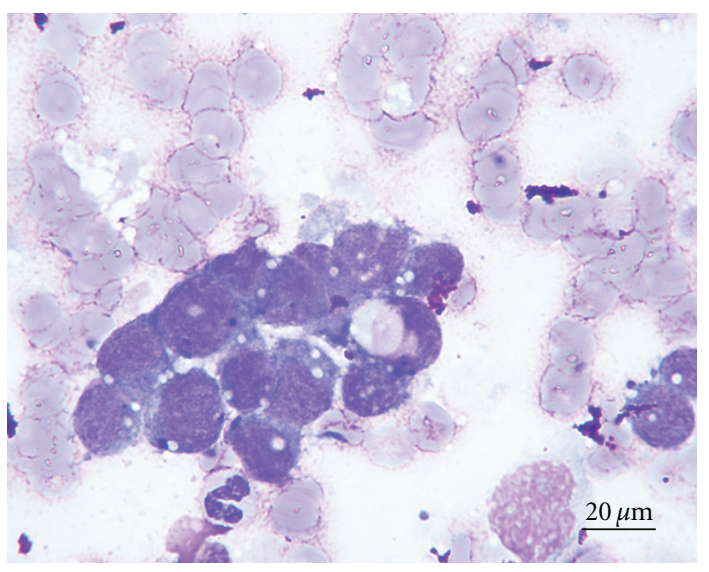

FIGURE 4: Fine-needle aspirate of an intra-abdominal mass in a young dog. Mildly pleomorphic round cells containing low numbers of vacuoles and one cell with a single large vacuole are present. These cells occasionally formed loose aggregates as seen here. The primary differential diagnosis was neoplasia (histiocytic sarcoma, other sarcomas, or atypical lymphoma). Wright's—Giemsa stain, $100 \mathrm{x}$ objective, bar $=20 \mu \mathrm{m}$.

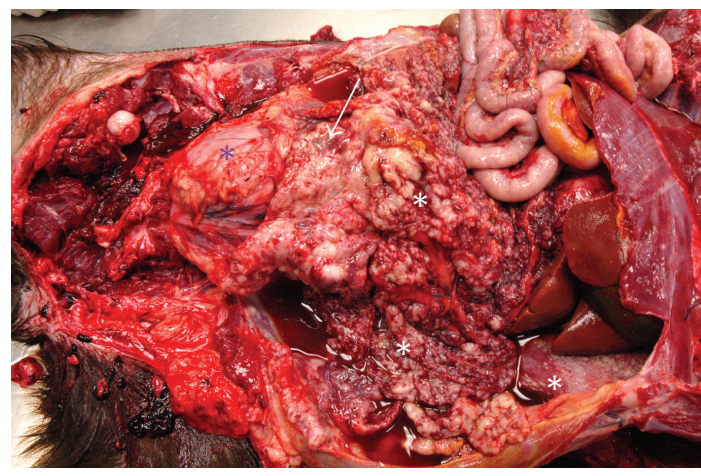

FIGURE 5: Gross image from the necropsy examination. Note the large abdominal mass (blue arrow) with extensive intra-abdominal dissemination (white asterisks: sarcomatosis). The urinary bladder is indicated by the blue asterisk.

further characterize these changes. However, it appears likely that the majority of tumor growth occurred after the last surgery which would explain why such widespread disease was not detected intraoperatively.

The patient's medical history and age influenced the interpretation of the fine-needle aspirate specimen. Because of the young age and multiple recent surgeries, caution was exercised when interpreting the cytology smears. Although neoplasia was the top differential diagnosis, chronic inflammation with marked cellular pleomorphism was deemed a reasonable consideration. Histopathology was considered necessary to further characterize the lesion.

With the exception of cutaneous histiocytomas of epidermal Langerhans cell origin, benign and malignant neoplasms of young dogs $(<2$ years $)$ are rare [1]. In one epidemiological study, only 117 out of the 60,000 cases (less than $0.2 \%$ ) of documented neoplasms over an eleven 
year period were in dogs less than 2 years of age [2]. In a similar study of immature dogs ( $<1$ year), neoplasms of the hematopoietic and central nervous system were the most frequently recognized types and site for neoplasia [3].

Sarcomas of all types are rare in young dogs, but the most frequently reported type is rhabdomyosarcoma. In young dogs, this tumor has been reported to arise in many tissues including the uterus $[1,4-7]$. In the previously mentioned survey of tumors in dogs less than 1 year of age, hemanigosarcoma and osteosarcoma were the most frequently recognized sarcomas with only one case of an undifferentiated sarcoma reported [3]. Interestingly, rhabdomyosarcomas were not reported in this study; however, this tumor has primarily been described in dogs over 1 year of age $[1,5-7]$.

Poorly differentiated or undifferentiated sarcomas can be defined as primitive mesenchymal tumors that cannot be readily classified to a specific lineage due to lack of specific morphologic, immunohistochemical, or genetic abnormalities [8]. Although continued advances in cytogenetics and immunohistochemical analysis are allowing the lineage of many tumors previously classified as undifferentiated sarcomas to be identified, sarcomas that cannot be further classified still exist. Whether these tumors represent a single tumor type or a mix of primitive mesenchymal tumors is still uncertain. [8]. Likewise, it is unclear whether the primitive nature of these tumors represents dedifferentiation or lack of/arrested differentiation of an undifferentiated cell type; however, research in the area of cancer stem cells implicates arrested differentiation as a mechanism in some primitive tumors [9-11].

Poorly differentiated sarcomas in young dogs have been rarely reported in the literature. One report of an aggressive, undifferentiated sarcoma with metastasis in a young dog has been previously described [12]. This sarcoma occurred in the cervical neck region with metastasis to organs including the lungs, liver, and kidneys. In another report, a seven-month old dog was diagnosed with an anaplastic subcutaneous sarcoma with widespread multiorgan metastasis [13]. No reports of a uterine/cervical poorly differentiated sarcoma in a young dog could be found.

Immunohistochemical analysis (vimentin positive) together with the gross and histologic appearance of the tumor was consistent with a diagnosis of sarcoma, but failed to further characterize the tumor type. Cytologically, histiocytic sarcoma was a differential, but lack of CD18 and lysozyme staining did not support this diagnosis. Muscle origin was also unsupported; cells were negative for multiple markers of both early (desmin and actin) and late (myoglobin) myocyte differentiation. Although the histopathologic appearance in conjunction with the immunohistochemistry results supports a clinical designation of poorly differentiated sarcoma, it is possible that with advanced techniques in immunohistochemical staining or electron microscopic evaluation of the tumor, a specific tumor lineage could have been identified [8, 14]. One example of advanced immunohistochemical staining techniques involves detection of myogenic regulatory protein (MyoD1), a sensitive indicator of early cell commitment to the myogenic cell line, which has been used to identify desmin negative embryonic rhabdomyosarcomas in people. This marker has also been used to characterize rhabdomyosarcomas in dogs [15]. Unfortunately, these advanced diagnostic tools were not available for this case.

In conclusion, sarcomas of any type are rare in young dogs and only sporadic cases of sarcomas that could not be further classified (poorly differentiated) have been reported. This patient was diagnosed with an aggressive, widespread, poorly differentiated sarcoma of uterine or cervical origin. Although unproven, this sarcoma may have been present at the time of the initial and/or subsequent surgeries. Pyometra is a common medical disease, and, paired with her young age, neoplasia was an unlikely cause for her initial and subsequent clinical signs. Nonetheless, this case highlights the importance of submitting abnormal tissue for histopathological examination despite clinical impression of disease. Regardless of when this sarcoma developed, it was aggressive in nature, as evidenced by the widespread organ metastasis.

\section{References}

[1] B. J. Cooper and B. A. Valentine, "Tumors of muscle," in Tumors in Domestic Animals, D. J. Meuten, Ed., pp. 319-363, Iowa State Press, Ames, Iowa, USA, 4th edition, 2002.

[2] D. Cohen, J. S. Reif, R. S. Brodey, and H. Keiser, "Epidemiological analysis of the most prevalent sites and types of canine neoplasia observed in a veterinary hospital," Cancer Research, vol. 34, no. 11, pp. 2859-2868, 1974.

[3] E. T. Keller and B. R. Madewell, "Locations and types of neoplasms in immature dogs: 69 cases (1964-1989)," Journal of the American Veterinary Medical Association, vol. 200, no. 10, pp. 1530-1532, 1992.

[4] J. Y. Yhee, D. H. Kim, D. N. Hwang et al., "Hematogenous metastasis of embryonal rhabdomyosarcoma originating from skeletal muscle in a young dog," Journal of Veterinary Diagnostic Investigation, vol. 20, no. 2, pp. 243-246, 2008.

[5] D. Y. Kim, E. C. Hodgin, D. Y. Cho, and J. E. Varnado, "Juvenile rhabdomyosarcomas in two dogs," Veterinary Pathology, vol. 33, no. 4, pp. 447-450, 1996.

[6] I. H. Bae, Y. Kim, B. Pakhrin et al., "Genitourinary rhabdomyosarcoma with systemic metastasis in a young dog," Veterinary Pathology, vol. 44, no. 4, pp. 518-520, 2007.

[7] O. G. Illanes, "Juvenile parameningeal rhabdomyosarcoma in a dog causing unilateral denervation atrophy of masticatory muscles," Journal of Comparative Pathology, vol. 126, no. 4, pp. 303-307, 2002.

[8] R. Alaggio, G. Bisogno, A. Rosato, V. Ninfo, and C. M. Coffin, "Undifferentiated sarcoma: does it exist? A clinicopathologic study of 7 pediatric cases and review of literature," Human Pathology, vol. 40, no. 11, pp. 1600-1610, 2009.

[9] S. Sell, "Cellular origin of cancer: dedifferentiation or stem cell maturation arrest?" Environmental Health Perspectives, vol. 101, no. 5, pp. 15-26, 1993.

[10] S. Sell and G. B. Pierce, "Maturation arrest of stem cell differentiation is a common pathway for the cellular origin of teratocarcinomas and epithelial cancers," Laboratory Investigation, vol. 70, no. 1, pp. 6-22, 1994.

[11] M. L. Suvà, N. Riggi, J. C. Stehle et al., "Identification of cancer stem cells in Ewing's sarcoma," Cancer Research, vol. 69, no. 5, pp. 1776-1781, 2009. 
[12] N. A. Sanders, R. L. Kerlin, and D. M. Dambach, "Aggressive, undifferentiated sarcoma with widespread metastasis in a six-month-old neopolitan mastiff," Journal of the American Animal Hospital Association, vol. 32, no. 2, pp. 97-101, 1996.

[13] G. Ghibaudo, G. Bettini, and F. Abramo, "Anaplastic and aggressive subcutaneous sarcoma in a seven-month-old dog," Journal of Small Animal Practice, vol. 49, no. 6, pp. 310-313, 2008.

[14] B. Madewell, J. Lund, R. Munn, and M. Pino, "Canine laryngeal rhabdomyosarcoma: an immunohistochemical and electron microscopic study," The Japanese Journal of Veterinary Science, vol. 50, no. 5, pp. 1079-1084, 1988.

[15] M. Murakami, H. Sakai, N. Iwatani et al., "Cytologic, histologic, and immunohistochemical features of maxillofacial alveolar rhabdomyosarcoma in a juvenile dog," Veterinary Clinical Pathology, vol. 39, no. 1, pp. 113-118, 2010. 

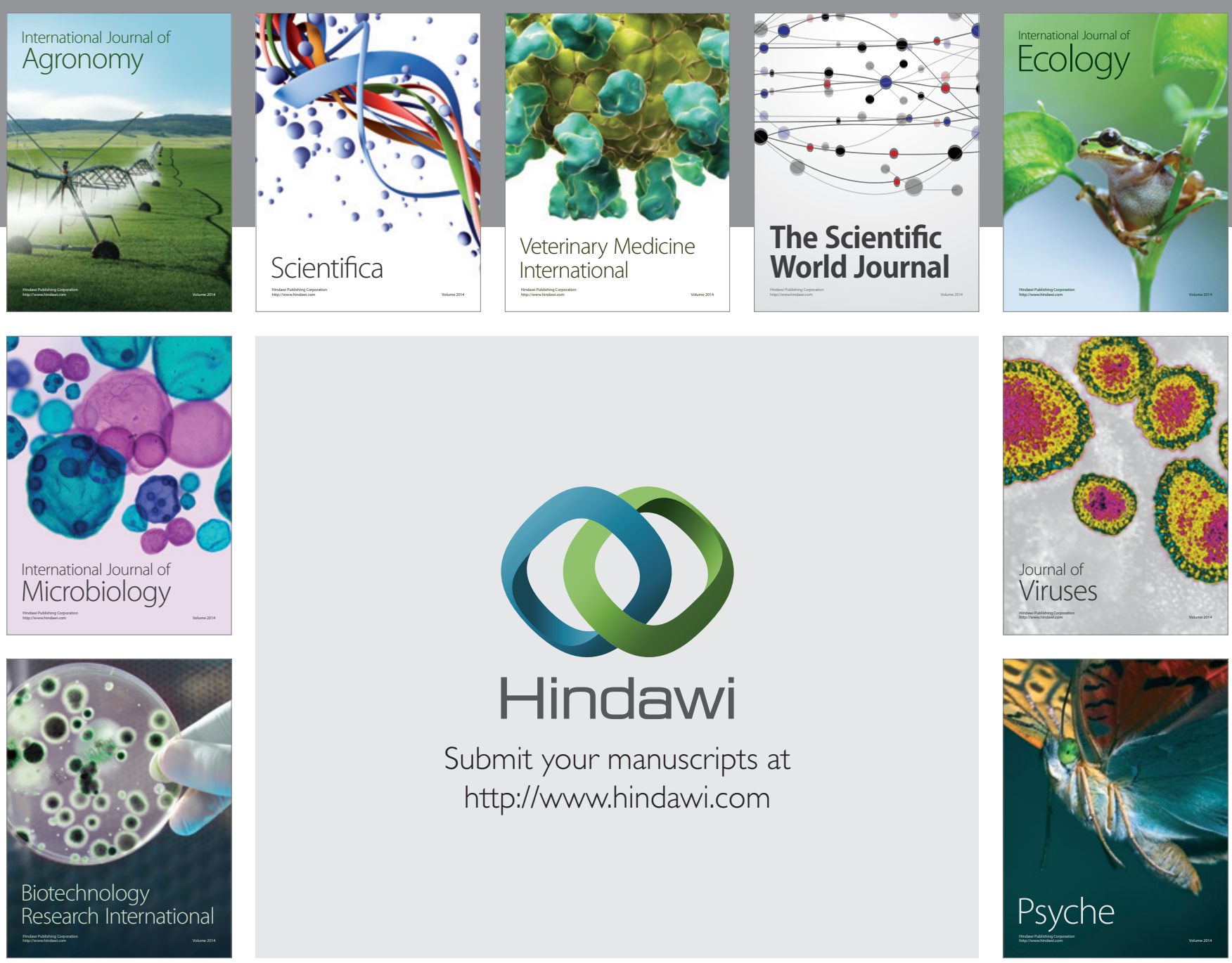

Submit your manuscripts at

http://www.hindawi.com
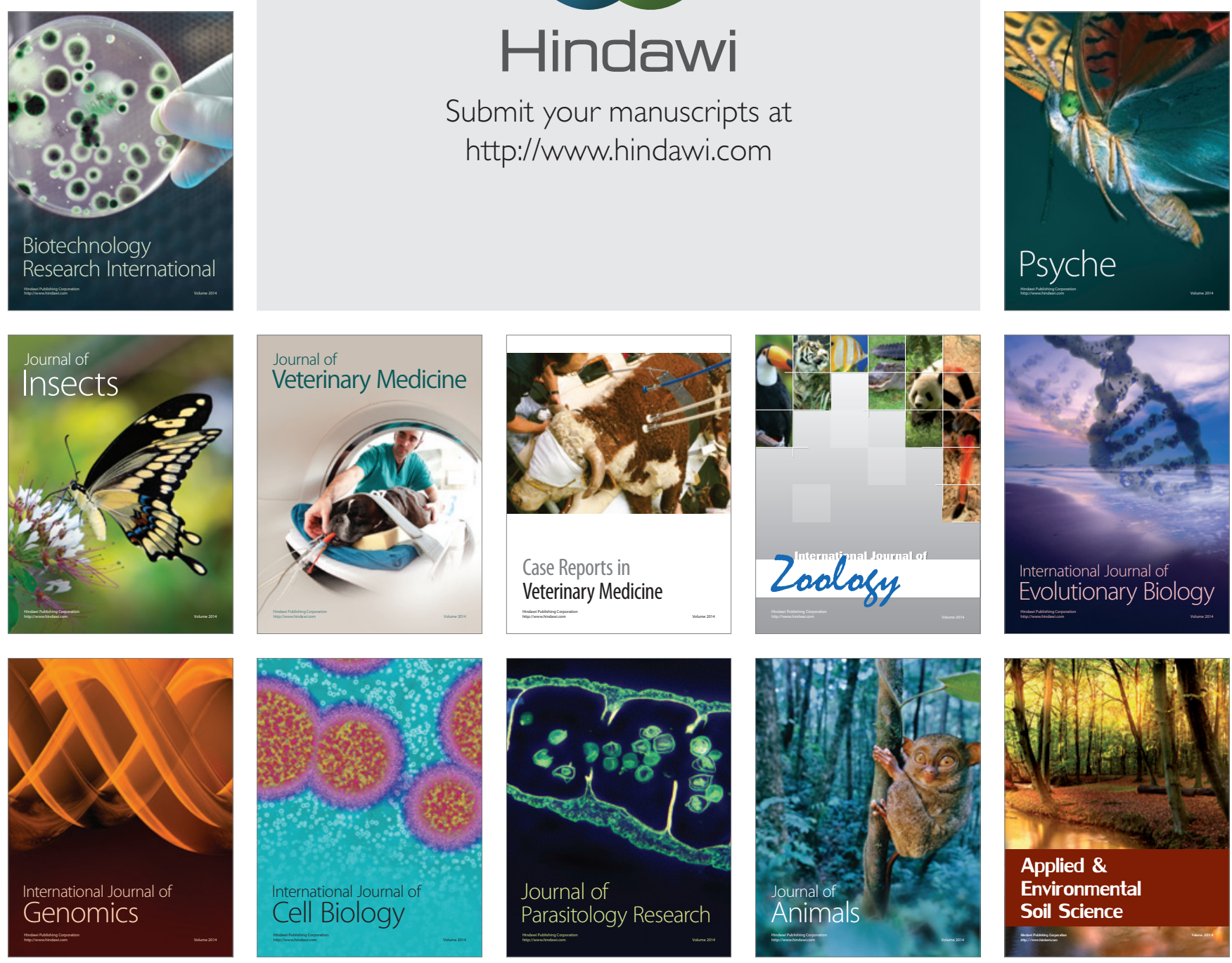